\title{
Identification of a target for CudA, the transcription factor which directs formation of the Dictyostelium tip organiser
}

\author{
HONG-YU WANG and JEFFREY G. WILLIAMS* \\ College of Life Sciences, University of Dundee, Dundee, U.K.
}

\begin{abstract}
The tip of the Dictyostelium slug functions much like an embryonic organiser; when grafted onto the flank of a recipient slug, it recruits a mass of prespore cells and leads them away as part of a secondary slug. CudA is a nuclear protein which is expressed in prespore cells where it acts as a specific transcription factor. CudA is also expressed in an anteriorly located group of cells, the tip-organiser, that is believed to constitute the functional tip. We identify an expansinlike gene, exp/7, that is expressed within the tip-organiser region and which is not expressed in a cudA null strain. The exp 17 promoter contains a region that binds to CudA in vitro and this region is necessary for expression in the tip-organiser. These results provide an end-point for a previously defined signal transduction pathway in which regionalised expression of the ACA adenylyl cyclase within the tip-organiser leads to localised CAMP-induced activation of STATa and consequent binding of STATa to the cudA promoter. STATa then induces expression of cudA and cudA directs the transcription of target genes such as expl7.
\end{abstract}

KEY WORDS: Dictyostelium, CudA, tip, expansin, gene expression

Cells within the tip of the Dictyostelium slug, that we will term the "tip-organiser" 1 , control the slug tissue in a manner analogous to vertebrate embryonic organisers. The tip-organiser also controls the choice between continued slug migration and immediate culmination (Smith and Williams, 1980). It is believed to play these roles by acting as a source of oscillatory cAMP production, signalling to cells behind it in the slug and controlling their behaviour. Consistent with such a notion, the ACA adenylyl cyclase, which is ubiquitously expressed during early development, becomes restricted in its expression to the tip-organiser (Verkerke-van Wijk et al., 2001). This triggers a number of signalling events, downstream from ACA, and they constitute a pathway leading to tip-organiser cell differentiation; signalling by the extracellular CAMP, that is produced locally within the tip-organiser, causes tyrosine phosphorylation of STATa so that STATa translocates into tip-organiser cell nuclei (Fukuzawa and Williams, 2000; Dormann et al., 2001). Then STATa binds to a dyad site within the cudA promoter and this induces cudA transcription (Fukuzawa and Williams, 2000).

The cudA gene was identified in a random mutagenesis screen as being essential for correct culmination (Fukuzawa et al., 1997). CudA is present in the nuclei of a cone of cells at the extreme slug tip and is also present in prespore cell nuclei. The cudA null strain is defective in the expression of certain prespore genes, including the cotC spore coat protein gene (Fukuzawa et al., 1997). The cudA null strain is also defective in control of entry into culmination, a behavioural property that is controlled by the tip region (Smith and Williams, 1980). This defect is corrected if the cudA gene is expressed in the cudA null mutant under the control of a promoter fragment that selectively directs expression to the anterior of the prestalk region (Fukuzawa et al., 1997). This suggests that CudA has a crucial role in tip cell differentiation and constitutes the evidence that the cudA expressing cells correspond to the functionally defined tip-organiser cells. Because there are no informative homologues in the databases, the molecular function of CudA has until recently been obscure. However, it is now known that CudA directly regulates $\cot C$ prespore gene expression, by binding to a site in the $\cot C$ promoter (Yamada et al., 2008), and we present evidence that it plays a similar, transcriptional role in tip-organiser cell differentiation.

\footnotetext{
Abbreviations used in this paper: ACA, adenylyl cyclase A; CudA, culmination defective A; ECudA, Entamoeba CudA homologue; expl, expansin-like; STAT, signal transducers and activators of transcription.
}

\footnotetext{
*Address correspondence to: Dr. Jeffrey G. Williams. College of Life Sciences, University of Dundee, Dow St., Dundee DD1 5EH, U.K. e-mail: j.g.williams@dundee.ac.uk
}

Note 1: http://dictybase.org/Dicty_Info/dicty_anatomy_ontology.html 
A

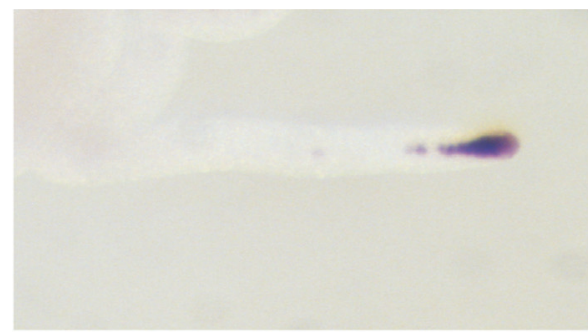

\section{AX2}

B

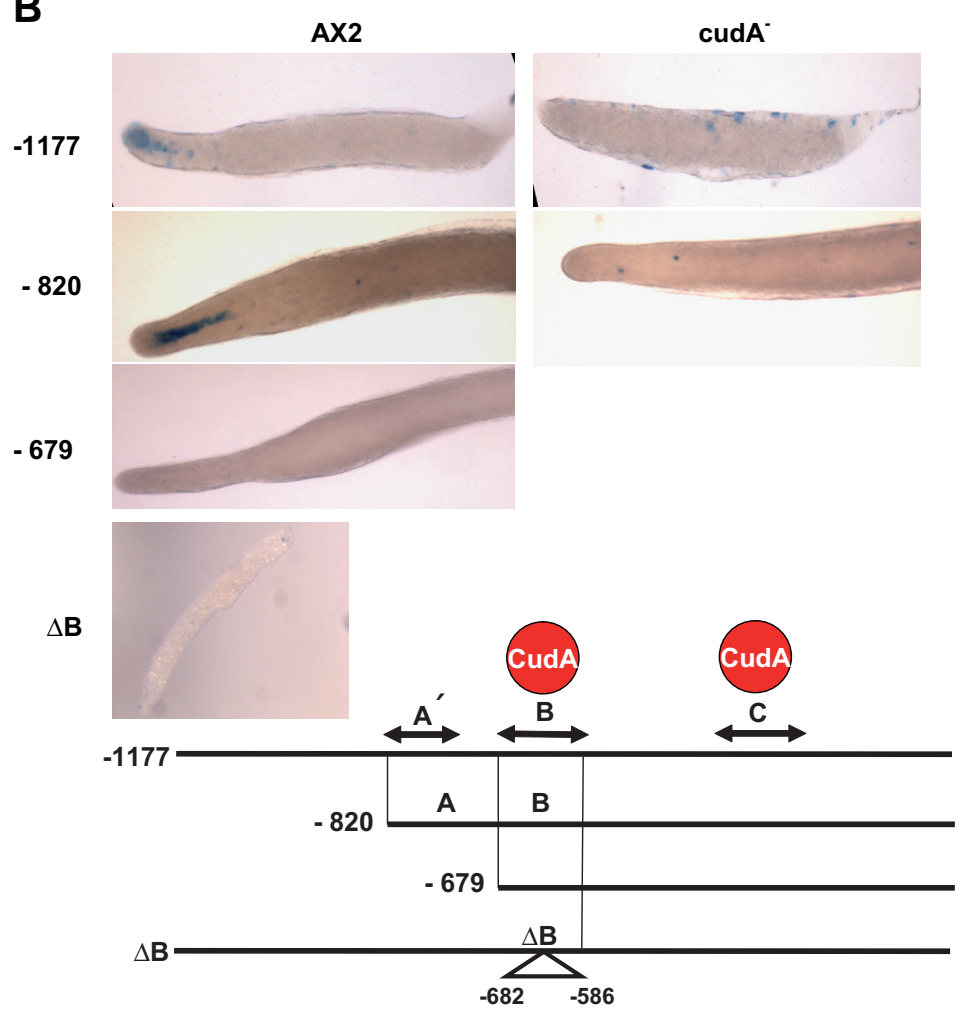

cudA*

Entire tip

Posterior tip

No expression

No expression

Fig. 1. Deletion analysis of the expl7 promoter. (A) In situ hybridisation of expl7 to parental and cudA null slugs. Parental (Ax2) and cudA null (cudA-) slugs were subjected to in situ hybridisation using an expl7 probe extending from the initiation codon to the stop codon. (B) Mutational analysis of the expl7 promoter. In all four constructs the lacZ gene is fused to expl7 at a point $51 \mathrm{bp}$ from the initiation codon, retaining the basal transcriptional signals and 5' non-coding region of expl7. Parental (Ax2) and, in some cases, cudA null (cudA-) cells (Yamada et al., 2008) were stably transformed with one of the indicated constructs and slugs were subjected to lacZ staining. As is typical of Dictyostelium promoters, the expl7 promoter contains long tracts of AT residues interspersed with more GC-rich regions. These GC-rich regions were used as anchor points for $P C R$ and the promoter sequences were scanned for binding of ECudA in a band shift assay, as in Fig 2B. As indicated by the red circles only two regions, $B$ and $C$, showed binding activity.

\section{Results}

\section{Expl7 $m R N A$ is expressed in the tip and is dependent upon cudA for its expression}

The starting point for this study was a set of prestalk-specific ESTs that were previously categorised by in situ-hybridisation at late developmental times (Maeda et al., 2003). We selected six (SLF308, SSK861, SLA128, SSL558, SSK348, SSB312) that seemed to most resemble the cudA expression pattern and determined their situhybridisation pattern in parental and cudA null slugs. Only SLA128, encoded by the exp/7 gene, has an expression pattern that matches that of the cudA mRNA (Fig 1A). Further consistent with a direct induction of exp/7 by CudA, there is no detectable expression of expl7 in cudA null slugs (Fig 1A).

The expl7 promoter can be sub-divided into regions that direct expression in different parts of the tip-organiser

An expl7 promoter fragment with a 5 endpoint at -1177 (numbered relative to the ATG initiation codon) was cloned upstream of lacZ and the construct was stably transformed into Ax2 and cudA null cells. In Ax2 cells the construct is expressed with a similar pattern to that observed by in situ hybridisation, suggesting that the entire promoter is present. Again, as in the in situ hybridisation analysis, there is no expression in the cudA null strain (Fig 1B). In order to narrow the search region for potential CudA binding sites, two 5 to $3^{\prime}$ deletion constructs were generated. Deletion from 1177 to -820 produces a change in the pattern of expression; staining is retained in the rear of the tip-organiser but is absent from the anterior region (Fig 1B). This presumably reflects a difference in spatial patterning between the two regions but we do not know the signalling basis for this difference. Expression within this foreshortened staining region is again dependent upon CudA. When the promoter is shortened further, to -679 , all expression is lost (Fig 1B). Thus essential elements for tip-specific expression are located between -820 and 679; we term this region $A$.

\section{A region downstream of the essential re- gion binds to CudA and is essential for tip- specific expression}

A sub-region of $A$, termed $A^{\prime}$ (the remainder of region $A$ is so AT-rich we elected not to analyse it) and region $B$, derived from promoter sequences downstream of -820 (Fig $1 \mathrm{~B})$, were used in affinity chromatography with slug stage nuclear extracts. The eluates were analysed by western transfer using a CudA monoclonal antibody. Region $B$ binds CudA but region $A^{\prime}$ does not (Fig 2A). We therefore generated an internal deletion construct, $\Delta \mathrm{B}$, that contains the entire promoter except for region $B$ and analysed its expression. It shows no activity (Fig 1B). Therefore, expression in the tip requires sequences located both in regions $A$ and $B$ and region $B$ contains one or more CudA binding sites. 


\section{ECudA binds to multiple dispersed sites within fragment $B$}

In order to identify potential CudA binding sites within fragment $B$ we performed band shift analysis. This necessitated using a recombinant protein; because there are six Dictyostelium CudA homologues that confuse the band shift assay and, for reasons that we do not understand, recombinant CudA is not active in DNA binding (Yamada et al., 2008). Hence we used a recombinant form of the Entamoeba CudA homologue, ECudA, that binds to the same site within the cotC promoter as CudA itself (Yamada et al., 2008). The probe in the band shift assays is a 66-mer from within the cotC promoter. As expected from the affinity chromatography results (Fig $2 A)$, fragment $B$ is able to compete efficiently with the 66-mer for binding to ECudA while fragment $A^{\prime}$ is not active (Fig 2B). This reinforces previous data, established using the cotC promoter, showing that CudA and ECudA have similar DNA binding properties (Yamada et al., 2008). ECudA also binds to a region, termed $C$, that is located between -345 and -221 (Fig 2B) but we did not study this region further. Instead we concentrated our efforts on mapping the site(s) within fragment $B$.

We first scanned the sequence for close matches to the ECudA binding site previously identified within the cotC promoter, GAAttTTC, but there are none (Fig 3). There are, however, four half sites (TTC): two very near the cap-site distal end, a third 41 nucleotides downstream of the first site and a fourth a further 30 nucleotides downstream. When a cap-site distal region containing two half-sites is deleted, to yield BD1, there is a partial reduction in competition efficiency (Fig 3). When a region containing the cap-site proximal half-site is de-

B
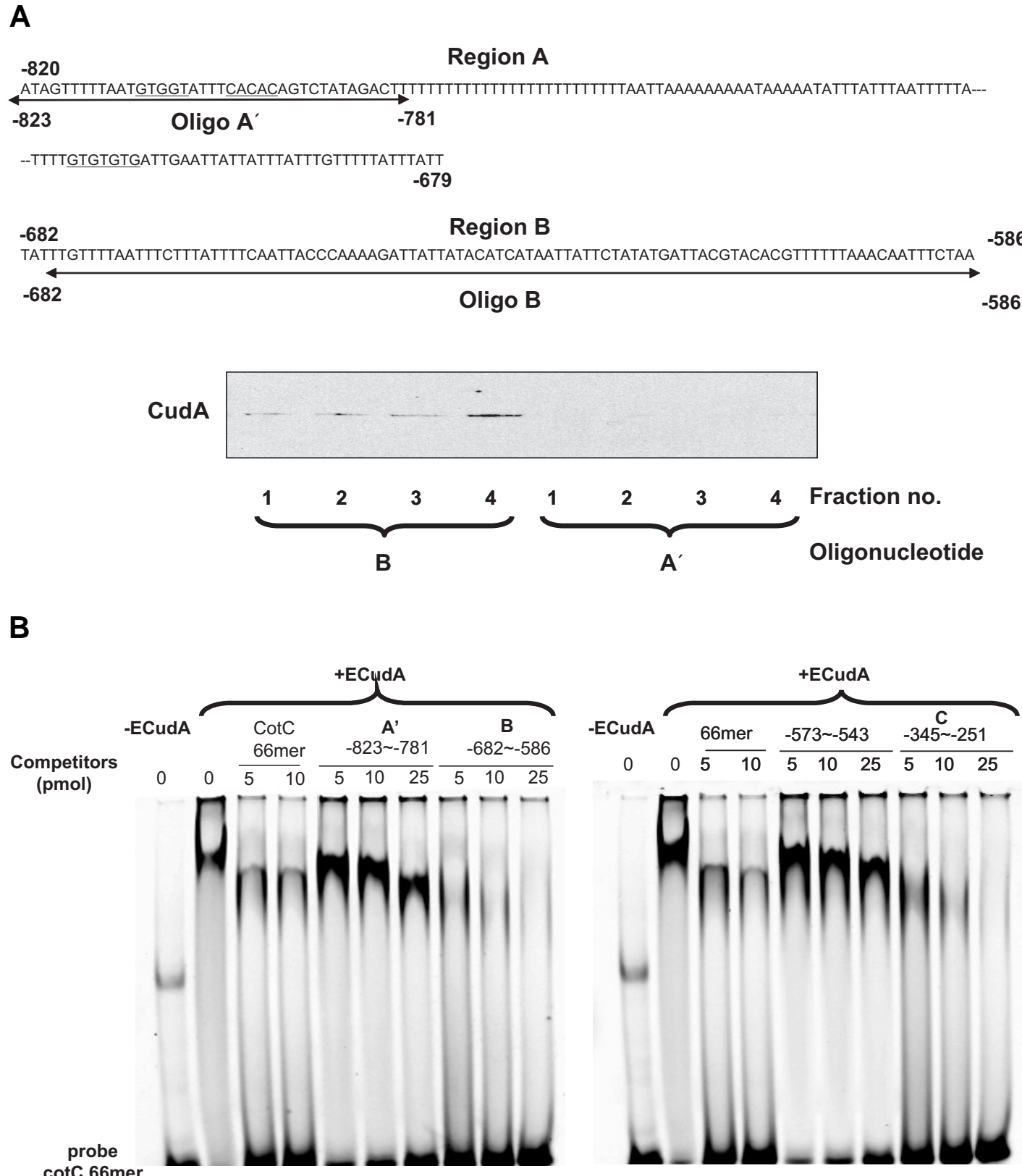

Fig. 2. Interaction analysis of CudA and ECudA with the expl7 promoter. (A) Analysis of the binding of CudA to oligonucleotides derived from the expl7 promoter Oligonucleotides $A^{\prime}$ and $B$, from regions $A$ and $B$ and with the sequences indicated by the double headed arrows, were used in affinity chromatography with Dictyostelium nuclear extracts. The bound protein was eluted in four fractions and analysed by Western transfer for CudA (Yamada et al., 2008). (B) Band-shift analysis of the binding of ECudA to oligonucleotides derived from the expl7 promoter. Recombinant ECUdA protein was used in band shift with a 66 nucleotide probe from within the promoter of the $\cot C$ gene (Yamada et al., 2008). In the absence of any competitor most of the probe is retarded by binding to ECudA. Oligonucleotide $B$ is at least as effective as the cotC 66-mer as a competitor for binding while oligonucleotide $A$ is almost ineffective at the concentrations employed. The gel at the right analyses a similar experiment using region $C$ and the indicated control, non CudA binding region as probes.

leted, to yield BD2, there is also a reduction in competition efficiency. Thus deletion from either end of region $B$ reduces competition efficiency. The BD2 construct contains the centrally located half-site and the proximal site and it retains competition activity. When, however, the centrally located half-site in BD2 is 


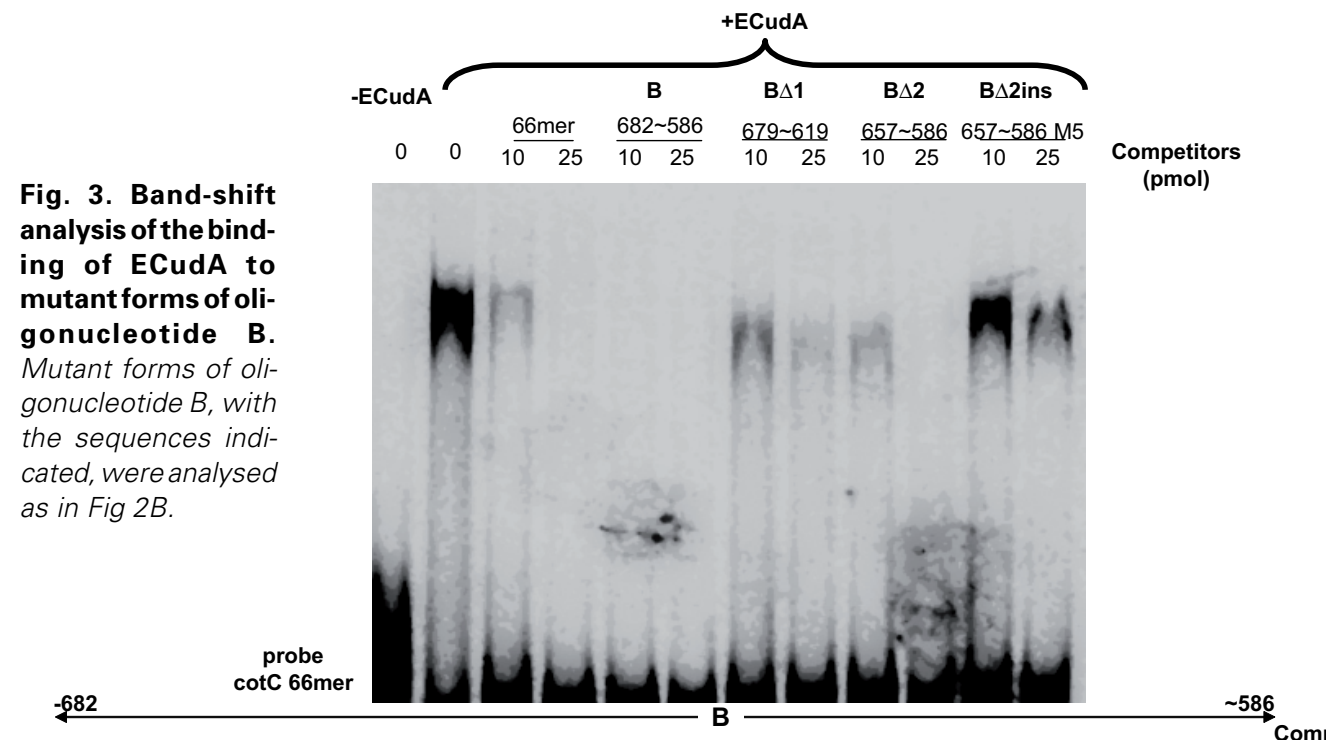

ATTTGTTTTAATTTCTTTATTTTCAATTACCCAAAAGATTATTATACATCATAATTATTCTATATGATTACGTACACGTTTTTTAAACAATTTCTAA +++

BA1 TGTTTTAATTTCTTTATTTTCAATTACCCAAAAGATTATTATACATCATAATTATTCTATA

$\mathrm{B} \Delta \mathbf{2}$

B $\Delta$ 2ins

limitation; prespore cells comprise $80 \%$ of the slug cells while tip cells comprise only a tiny proportion of the slug cell population, making signal detection problematical. Evidence for a direct interaction comes from analysis of the expl7 promoter.

Two essential regions, $A$ and $B$, were identified within the promoter and region $B$ displays CudA binding. The binding sites in region $B$ were approximately mapped, using a combination of deletion analysis and sequence replacement, and the regions identified each contain a potential half-site for CudA binding (TTC). The regions are, however, widely spaced and there is a separate CudA binding domain proximal to the cap site, within region $\mathrm{C}$. These facts complicate the biochemical and the funcanalysis greatly, hence we have not mapped the sites further. The fact that regions $A$ and $B$ are both essential for expression implies a co-operative interaction between

replaced with a CG rich sequence, to yield BD2ins, there is a very large reduction in competition efficiency. Thus mutation of three widely separated regions, each containing a TTC sequence, reduce ECudA binding. We therefore believe that there is cooperative binding of ECudA to the half-site sequences. This is in accord with our previous study where we showed that ECudA binds co-operatively to target sequences and presented in vivo evidence that a half-site augments the biological activity of CudA as an activator of prespore gene expression (Yamada et al., 2008).

\section{Discussion}

exp/7 encodes an expansin. Expansins are found in all groups of land plants, where they interact with cell wall components including cellulose, and they are also found in Dictyostelium (reviewed in (Darley et al., 2003)). The expression of exp/7 in tip cells presumably reflects the fact that, at culmination, tip cells enter the stalk tube and undergo major changes in cell wall structure that involve the deposition of cellulose. It will be of considerable interest to analyse the function of exp/7 genetically but this may be complicated by functional redundancy between members of the large gene family. The exp/7 gene has a similar pattern of expression to cudA and it is not expressed in a CudA null strain. Exp/7 is also not expressed in STATa null slugs (Shimada et al., 2004) and this is as expected if CudA is a positive transcriptional regulator of exp/7; because STATa directs the expression of cudA (Fukuzawa and Williams, 2000). ChIP analyses of the cotC prespore promoter indicate that regulation of transcription by CudA is due to its direct binding to the promoter (Yamada et al., 2008). We were unable to detect a signal in ChIP analysis of the exp/7 promoter using a CudA antibody (unpublished results) but we believe that this probably reflects a technical
CudA and another transcription factor. Such interactions have been shown to occur with GBF (Ceccarelli et al., 1992; PowellCoffman et al., 1994, Schnitzler et al., 1994), a zinc finger transcription factor. There are potential GBF binding sites, "G boxes" in region $A$ (underlined in Fig $2 A$ ) but heterologous, $G$ boxes, from the CotC promoter (-403 to -386) and from the ecmB promoter (-818 to -802$)$, could not subsume the function of region A (unpublished data).

One related, intriguing issue raised by these results is that of dual specificity; how is CudA able to function as a transcriptional activator in both the prespore and the tip-organiser regions? Presumably CudA interacts with different ancillary transcription factors in the two different cell populations. Given the predominant biological role of the tip-organiser, it will be important to identify such factors.

\section{Materials and Methods}

Cell culture, development, transformation and expression analysis All experiments were performed with the Gerisch isolate of Ax2 and cells were grown, developed and transformed as described previously (Fukuzawa and Williams, 2000). In situ hybridisation and lacZ expression analyses were also as described (Fukuzawa and Williams, 2000). Promoter fragments from exp/7 were generated by PCR and cloned into the vector pDd17Gal at the BamHI and Bglll sites. The internal deletion mutant was created by sequentially cloning two exp/7 fragments, -1177 to -677 and -573 to +50 into the pDd17Gal vector at its EcoRI and Bgll sites, resulting in a $103 \mathrm{bp}$ deletion.

\section{Analysis of CudA binding}

Slug nuclear extracts were obtained by sonicating nuclei in DB buffer (50mM KPO4, pH7.5, 10\% glycerol, 0.5mM EDTA, 0.1 mM ZnCl, $0.1 \mathrm{mM}$ $\mathrm{MgCl}_{2}, 0.01 \%$ Brij 35) containing $0.1 \mathrm{M} \mathrm{NaCl}, 2 \mathrm{mM}$ benzamidine hydrochloride, complete protein inhibitor cocktail, $10 \mathrm{mM}$ sodium fluoride and $1 \mathrm{mM}$ sodium pyrophosphate. The oligonucleotides were concatemarised 
and coupled to $\mathrm{CNBr}$-sepharose 4B. The slug nuclear extract was precleared with blocked $\mathrm{CNBr}$-sepharose $4 \mathrm{~B}$ and then incubated with sepharose $4 \mathrm{~B}$ bearing the oligonucleotide. After washing with $\mathrm{DB}$ buffer containing $0.1 \mathrm{M} \mathrm{NaCl}$, bound protein was eluted with $\mathrm{DB}$ buffer containing $0.4 \mathrm{M} \mathrm{NaCl}$. Protein was concentrated by precipitating with $13 \%$ TCA and analysed by Western blotting using an anti-CudA antibody.

\section{ECudA band shift analysis}

The entire ECudA coding region was cloned as A HIS-ECudA fusion construct (Yamada et al., 2008) in pET15b (Novagen, Ltd) was expressed and purified over a TALON ${ }^{\mathrm{TM}}$ metal affinity resin (BD Biosciences, Ltd). Band shift analysis was performed as described previously (Kawata et al., 1996) using oligonucleotides labelled with Cy5-dCTP (Amersham, Ltd). Gels were scanned at $700 \mathrm{~nm}$ wavelength with the Odyssey Infrared Imaging System (LI-COR, Ltd.).

\section{Acknowledgements}

We would like to thank the Wellcome Trust (Project Grant 078971/Z) 05/Z) for funding this work.

\section{References}

CECCARELLI, A., MAHBUBANI, H.J., INSALL, R., SCHNITZLER, G., FIRTEL, R.A. and WILLIAMS, J.G. (1992). A G-rich sequence element common to Dictyostelium genes which differ radically in their patterns of expression. Dev. Biol. 152: 188-193.

DARLEY, C.P., LI, Y., SCHAAP, P. and MCQUEEN-MASON, S.J. (2003). Expression of a family of expansin-like proteins during the development of Dictyostelium discoideum. FEBS Lett. 546: 416-418.
FUKUZAWA, M. and WILLIAMS, J.G. (2000). Analysis of the promoter of the cudA gene reveals novel mechanisms of Dictyostelium cell type differentiation. Development 127: 2705-2713.

MAEDA, M., SAKAMOTO, H., IRANFAR, N., FULLER, D., MARUO, T., OGIHARA S., MORIO, T., URUSHIHARA, H., TANAKA, Y. and LOOMIS, W.F. (2003). Changing patterns of gene expression in Dictyostelium prestalk cell subtypes recognized by in situ hybridization with genes from microarray analyses. Euk. Cell 2: 627-637.

POWELL-COFFMAN, J.A., SCHNITZLER, G.R. and FIRTEL, R.A. (1994). A GBFbinding site and a novel AT element define the minimal sequences sufficient to direct prespore-specific expression in Dictyostelium discoideum. Mol. Cell. Biol. 14: 5840-5849.

SCHNITZLER, G.R., FISCHER, W.H. and FIRTEL, R.A. (1994). Cloning and characterization of the G-box binding factor, an essential component of the developmental switch between early and late development in Dictyostelium. Genes Devel. 8: 502-514.

SHIMADA, N., MAEDA, M., URUSHIHARA, H. and KAWATA, T. (2004). Identification of new modes of Dd-STATa regulation of gene expression in Dictyostelium by in situ hybridisation. Int. J. Dev. Biol. 48: 679-682.

SMITH, E. and WILLIAMS, K. (1980). Evidence for tip control of the «slug/fruit» switch in slugs of Dictyostelium discoideum. J. Embryol. Exp. Morphol. 57: 233 240.

VERKERKE-VAN WIJK, I., FUKUZAWA, M., DEVREOTES, P.N. and SCHAAP, P (2001). Adenylyl cyclase A expression is tip-specific in Dictyostelium slugs and directs StatA nuclear translocation and CudA gene expression. Dev. Biol. 234: 151-160.

YAMADA, Y., WANG, H. Y., MASASHI, F., BARTON, G. J. AND WILLIAMS, J. G. A new family of transcription factors Development 135: 3093-3101.

\section{Further Related Reading, published previously in the Int. J. Dev. Biol.}

See our recent Special Issue Epigenetics \& Development edited by Saadi Khochbin and Stefan Nonchev at: http://www.ijdb.ehu.es/web/contents.php?vol=53\&issue=2-3

Expression of zinc transporter family genes in Dictyostelium Nobuya Sunaga, Meri Monna, Nao Shimada, Mai Tsukamoto and Takefumi Kawata Int. J. Dev. Biol. (2008) 52: 377-381

Analysis of a homologue of the adducin head gene which is a potential target for the Dictyostelium STAT protein Dd-STATa

Ryota Aoshima, Rieko Hiraoka, Nao Shimada and Takefumi Kawata Int. J. Dev. Biol. (2006) 50: 523-532

Identification of new modes of Dd-STATa regulation of gene expression in Dictyostelium by in situ hybridisation Nao Shimada, Mineko Maeda, Hideko Urushiharaand Takefumi Kawata Int. J. Dev. Biol. (2004) 48: 679-682

Expression and role of adenylyl cyclases during late development in Dictyostelium discoideum

E Alvarez-Curto, ME Meima, P Schaap

Int. J. Dev. Biol. (2001) 45: S147-S148

Dictyostelium discoideum: a model system for differentiation and patterning. $\mathrm{R}$ Escalante and $\mathrm{J} \mathrm{J}$ Vicente Int. J. Dev. Biol. (2000) 44: 819-835

5 yr ISI Impact Factor $(2008)=3.271$

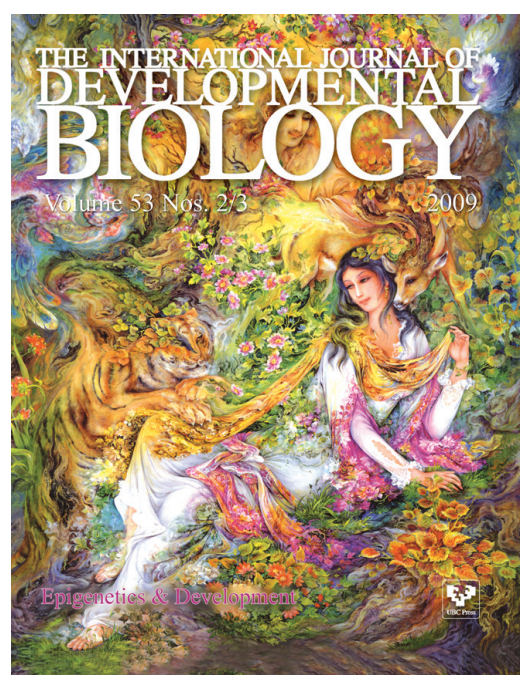

